\title{
MCERL y vocabulario receptivo de estudiantes españoles de Francés como Lengua Extranjera
}

\author{
Guadalupe DE LA MAYA RETAMAR \\ Universidad de Extremadura \\ gmaya@unex.es \\ https://orcid.org/0000-0003-4201-4024
}

\author{
Inmaculada MORA RAMOS \\ Universidad de Extremadura \\ imorar06@educarex.es \\ https://orcid.org/0000-0002-4172-1459
}

\section{Resumen}

La investigación que presentamos se plantea el doble objetivo de determinar el tamaño del vocabulario receptivo de estudiantes de francés de Escuela Oficial de Idiomas y establecer una correspondencia con los niveles del Marco común europeo de referencia para las lenguas (MCERL). La muestra, constituida por 50 estudiantes que cursan los niveles A1-A2-B1-B2, completó un test de vocabulario receptivo, cuyo análisis nos permitió determinar el tamaño de sus vocabularios, valorar el progreso de un nivel al siguiente y estudiar la relación entre dicha extensión y los distintos niveles. Los resultados nos permiten concluir que el vocabulario es un indicador del nivel de competencia y que la obtención de un determinado nivel está en dependencia del tamaño del vocabulario.

Palabras clave: vocabulario, francés como lengua extranjera, extensión vocabulario receptivo, MCERL.

\section{Résumé}

Dans cet article, nous présentons les résultats d'une étude menée avec des étudiants de FLE d'une École Officielle de Langues. L'objectif a été double, d'un côté, déterminer la taille de leur vocabulaire réceptif, de l'autre, établir une correspondance entre celui-ci et les niveaux du Cadre européen commun de référence pour les langues (CECRL). L'analyse d'un test effectué par un échantillon de 50 étudiants nous a permis d'établir la taille de leur vocabulaire, de mesurer les progrès d'un niveau à l'autre et d'étudier la relation entre cette extension du vocabulaire et les différents niveaux. Les résultats nous permettent d'affirmer que le vocabulaire réceptif est un indicateur du niveau de compétence et que l'obtention d'un certain niveau du CECRL dépend de la taille du vocabulaire.

Mots-clés : vocabulaire réceptif, taille du vocabulaire, FLE, CECRL.

* Artículo recibido el 11/07/2020, aceptado el 28/12/2020. 


\begin{abstract}
Research on the receptive vocabulary of languages learners highlights his relationship with the general competence in languages. In this line, some studies have been carried out in order to associate the size of the vocabulary and the levels of the Common European Framework (CEFRL). We present the results of a research developed with French students in an Official School of Languages, with the double objective of determining the size of their receptive vocabulary and establishing a correspondence with the levels of the CEFRL. We conclude that the vocabulary is an indicator of the level of competence, and that obtaining a certain level is dependent on the size of the vocabulary.
\end{abstract}

Keywords: vocabulary, French as foreign language, languages teaching, CEFRL.

\title{
1. Introducción
}

Si bien a partir de la pasada década de los 80 , los estudios sobre vocabulario experimentaron un gran auge que permitía olvidar la condición de pariente pobre con la que Galisson (1971) calificaba al vocabulario en el contexto del aprendizaje de lenguas, no cabe duda de que, aún hoy en día, hay muchas cuestiones sobre las que profundizar. Esa década marca un punto de inflexión, pues en los últimos cuarenta años se han sucedido numerosos estudios (Arnaud y Béjoint, 1992; Bogaards y Laufer, 2004; Coady y Huckin, 1997; Daller, Milton y Treffers-Daller, 2007; Meara, 2009; Nation, 1990, 2001, 2016; Schmitt, 2010), especialmente sobre el inglés como lengua extranjera, que intentan arrojar luz sobre la adquisición, el desarrollo y la evaluación del vocabulario en segundas lenguas, así como sobre aquellos aspectos, individuales o contextuales que pueden condicionarlo. También, en francés como lengua materna y extranjera, hemos asistido a la publicación de monografías (Bogaards, 1994; Calaque y David, 2004; Grossmann, Paveau y Petit, 2005; Tréville, 2000; Tréville y Duquette, 1996) y númerosos monográficos de revistas (Journal of French Language Studies, 18: 3, 2008; La revue canadienne des langues vivantes, 53, 1996; 63: 1, 2006; Acquisition et Interaction en Langue étrangère, 3, 1994; LIDIL, 21, 2000; Études de Linguistique Appliquée, 116, 1999; Les Langues Modernes, 1, 2009; Le français dans le monde. Recherches et Applications, 1989) que han facilitado que la didáctica del léxico despierte del sueño en que estaba sumida, tal y como plantean Grossman et al. (2005) en el prólogo de su trabajo.

No obstante, a pesar de que todas estas monografías y estudios han contribuido a aumentar la eficacia de la enseñanza y aprendizaje del vocabulario, la investigación en adquisición del vocabulario en segundas lenguas se caracteriza por un nivel elevado de fragmentación y por la ausencia de resultados concluyentes (Jiménez y Terrazas, 20052008), de ahí que se planteen aspectos del vocabulario que requieren de nuevas investigaciones en este campo (Jiménez y Moreno, 2004-2005): desarrollo y progreso en el 
aprendizaje de nuestros estudiantes, comparación del tamaño de sus vocabularios, selección del input léxico, niveles de vocabulario en relación con las etapas educativas o evaluación del desarrollo, tamaño del vocabulario y logros alcanzados por los alumnos. A pesar de que las investigadoras aludidas realizaban esta propuesta teniendo en cuenta los niveles de Educación Primaria y Secundaria, no podemos olvidar que, en la Ley que rige nuestro sistema educativo, está regulada una enseñanza de idiomas incluida en las enseñanzas de régimen especial, por lo que creemos que ese planteamiento puede y debe ser también extensible al alumnado de Escuelas Oficiales de Idiomas (EOI), centros sobre los que se articula ese tipo de enseńanza. Y pensamos que algunas de esas necesidades investigadoras son especialmente aplicables a la enseñanza de idiomas de las EOI, si tenemos en cuenta que la formulación de los contenidos léxico-semánticos necesarios para la superación de cada uno de los niveles en los que se estructura esta enseñanza (básico, intermedio y avanzado) tiene en los currículos una formulación genérica en la que únicamente se plantea que el léxico se adecuará a los objetivos especificados para cada nivel.

A pesar del aumento, ya apuntado, de investigaciones en el terreno del vocabulario, especialmente en el contexto español (Agustín y Cangas, 2016; Agustín y Terrazas, 2009, 2012; Fernández, 2017; Jiménez, 2010, 2014; Jiménez y Canga, 2019; Jiménez y Moreno, 2004-2005; Jiménez y Terrazas, 2005-2008), si nos centramos en el ámbito del francés como lengua extranjera, una revisión de la literatura (De la Maya Retamar, 2015; De la Maya y Mora, 2019; Batista y Horst, 2016; Blais, 2014; David, 2008; Greidanus, Beks y Wakely, 2006; Greidanus y Nienhuis, 2001; Lindqvist, Bardel y Gudmundson, 2001; Milton, 2006, 2008; Ovtcharov, Cobb y Halter, 2006; Richards, Malvern y Graham, 2008; Tibdall y Treffers-Daller, 2008) nos permite comprobar la poca información de la que disponemos acerca del vocabulario que aprenden nuestros estudiantes, debido fundamentalmente a la escasez de investigación, pues la que se ha realizado ha tomado como lengua de estudio el inglés como lengua extranjera, a la falta de interés que esta cuestión parece despertar entre los investigadores, especialmente en el contexto español, y al número limitado de cuestiones acerca del vocabulario que se tratan, pues el foco reside casi exclusivamente en el análisis del tamaño del vocabulario y su desarrollo.

Así pues, el objetivo de este trabajo es paliar, en parte, esa ausencia de investigaciones, analizando el tamaño del vocabulario receptivo de estudiantes de Escuela Oficial de Idiomas, su desarrollo a lo largo de diferentes cursos y la definición de niveles de dominio asociados a ellos. Nos centramos en el vocabulario receptivo, que implica reconocer oralmente o por escrito la forma de una palabra y recuperar su significado (Nation, 2001), dado que disponemos de test validados para su evaluación, aspecto que con el vocabulario productivo resulta problemático. Analizamos el tamaño del vocabulario y su desarrollo porque consideramos con Jiménez y Terrazas (2005-2008) que este aspecto permitirá cuantificar lo que los estudiantes han aprendido cada curso y, 
consecuentemente, ajustar la planificación para solventar posibles lagunas. Finalmente situamos nuestra investigación en el contexto de la enseñanza de idiomas de régimen especial por dos razones fundamentales: de un lado, porque este alumnado no ha sido, que sepamos, objeto de investigaciones previas; de otro, porque es nuestro interés asociar el vocabulario con los niveles del Marco común de referencia para las lenguas (Consejo de Europa, 2002) y es en este tipo de enseñanza en la que se hallan fijados los niveles de dominio que deben alcanzarse en los distintos cursos en los que se estructura.

\section{Fundamentación teórica}

El estudio de la competencia léxica puede enfocarse desde perspectivas diferentes (Öztürk, 2003): lo que sabemos acerca de las palabras, es decir, sus componentes, cómo conocemos las palabras o cuántas y qué palabras se conocen. Puesto que el objeto de estudio de este trabajo es el tamaño del vocabulario receptivo, nos centraremos en la segunda y tercera de estas perspectivas, analizando el grado variable de conocimiento léxico (Laufer, Elder, Hill y Congdon, 2004; Read, 2000) por una parte, y el número de palabras que son conocidas por un hablante, por otra.

\subsection{El vocabulario receptivo}

La distinción entre conocimiento receptivo y productivo es entendida por algunos autores como una dicotomía (Clark, 1993; Meara, 1990), lo que implica que estaríamos ante dos conceptos, vocabulario receptivo (pasivo) y productivo (activo), cualitativamente diferentes, pues mientras el primero solo reacciona a estímulos externos, es decir, lo reconocemos cuando lo vemos o lo escuchamos, pero no lo recordamos sin ese soporte externo, el vocabulario activo no requiere de estímulos externos, pudiendo ser activado por otras palabras (Meara, 1990). Sin embargo, otros autores, como Melka (1997), abogan por una visión de dicho conocimiento como un continuo, considerando el conocimiento como un único sistema que puede ser utilizado de dos maneras distintas, receptivamente o productivamente.

Nation (2001), en su propuesta sobre lo que implica conocer una palabra, toma en consideración el uso receptivo y productivo de la palabra, distinción que se basa, a su vez, en la efectuada entre las destrezas receptivas y las productivas. Mientras que el término receptivo transmite la idea de que somos nosotros los que recibimos lenguaje de otras personas a través de actividades de escucha o lectura y tratamos de comprender, productivo, por su parte, indica que producimos lenguaje a través de tareas de expresión, sea oral o escrita, con el fin de transmitir mensajes a los demás:

[...] receptive vocabulary use involves perceiving the form of a word while listening or reading and retrieving its meaning. Productive vocabulary use involves wanting to express a meaning through speaking or writing and retrieving and producing the appropriate spoken o written word form (Nation, 2001: 25-26). 
Nation (2001) aplica, además, esta distinción al conocimiento (forma y significado) y al uso léxico, planteando una lista de preguntas en relación con dichos conocimientos que deberíamos ser capaces de responder para considerar que se conoce una palabra (significado, forma escrita y oral, comportamiento gramatical, colocaciones, registro, asociaciones y frecuencia) y mostrando la riqueza y complejidad de este concepto. No obstante, como señala Milton (2013), con el objeto de reducir dicha complejidad, ha sido frecuente pensar en el conocimiento léxico más en término de dimensiones que en una lista de elementos discretos. Cada dimensión podría abarcar todos los elementos de la lista de Nation (2001) vinculados entre sí, formado una entidad única y más grande. En este sentido, el tamaño o la extensión del vocabulario, es decir, el número de palabras que un hablante conoce, ha sido una de las dimensiones más abordadas en la investigación acerca de este tema en segundas lenguas (Gyllstad, 2013), siendo considerada esencial por parte de numerosos autores. Así, Meara (1996: 37) determina que:

All others things being equal, learners with big vocabularies are more proficient in a wide range of language skills than learners with smaller vocabularies, and there is the view that vocabulary skills make a significant contribution to almost all aspect of L2 proficiency.

Efectivamente, muchos son los estudios que han relacionado esta dimensión con la competencia general en lenguas, poniendo de manifiesto que cuanto mayor es el vocabulario del aprendiz, más competente es en la lengua en cuestión (Alderson, 2005; Fan, 2000; Hilton, 2007; Milton, 2010; Milton, Wade y Hopkins, 2010; Nasir, Manan y Azizan, 2017; Ovtcharov et al., 2006; Zareva, Schwanenflugel y Nikolova, 2005). Así, por ejemplo, Fan (2000) en su estudio realizado con estudiantes chinos que se incorporaban a la universidad, sugiere, tras analizar los resultados obtenidos por dichos alumnos en un test de vocabulario receptivo y su puntuación alcanzada en inglés en las pruebas completadas para su acceso a la enseñanza superior, que son los estudiantes más competentes en inglés aquellos que tienen un vocabulario receptivo mayor.

Asimismo, también la relación entre el tamaño del vocabulario receptivo y las distintas destrezas ha sido objeto de estudio, señalándose la correlación existente entre aquel y la comprensión escrita, la expresión escrita o la comprensión oral (Agustín y Terrazas, 2009; Harkio y Pietilä, 2016; Laufer, 1992; Milton et al., 2010; Qian, 2002; Staehr, 2008). Los resultados ponen de manifiesto la importancia de que los alumnos cuenten con un vocabulario extenso, aspecto que resulta ser clave si el objetivo es una comunicación efectiva en lengua extranjera. Esta es la razón que ha guiado numerosos trabajos de investigación cuyo objetivo ha sido establecer un umbral léxico, es decir, cuantificar el número mínimo de palabras que permita realizar diversas tareas comunicativas, tales como comprender un texto oral o escrito. Si bien sobre el inglés se han 
realizado diferentes estudios (Adolphs y Schmitt, 2004; Laufer, 1989; Laufer y Ravenhorst-Kalovski, 2010; Nation, 2006 o Staehr, 2008), sobre el francés no conocemos más que el trabajo de Cobb y Horst (2004). Los resultados muestran las diferencias existentes entre las lenguas. Así, si para alcanzar un alto nivel de cobertura de un texto escrito -aproximadamente el 85\%-, en inglés es necesario conocer las 2000 familias de palabras más frecuentes más las 570 familias de palabras recogidas en la Academic Word List (Coxhead, 2000), para el francés ese nivel de cobertura es factible con solo conocer las 2000 familias de palabras más frecuentes:

[...] the French 2000 list seems to serve both everyday and academic purposes more effectively than its English counterpart, such that there appears to be no need for an additional AWLlike list in French to facilitate the comprehension of academic texts. With the coverage powers of the French 2000 list so high, there appears to be little or no space left in the lexis of French for such a list to occupy (Cobb y Horst, 2004: 15).

\subsection{El tamańo del vocabulario receptivo y su relación con el Marco común europeo de referencia para las lenguas}

Como señala Milton (2010: 218), la relación entre el tamaño del vocabulario y la competencia general en lenguas, de un lado, y las distintas destrezas, de otro, aspectos presentados en el apartado anterior, revelan la relación que existe entre la extensión del vocabulario receptivo y el nivel de competencia y habilidad que el alumno consigue: "High level performers tend to have extensive vocabulary knowledge and elementary level performers do not".

Esta relación ha motivado la realización de determinados trabajos desarrollados con el fin de asociar el tamaño del vocabulario de los aprendices y los distintos niveles de competencia alcanzados por ellos (Meara y Milton, 2003; Milton y Alexiou, 2009; Milton, 2013). En unos casos, los niveles de competencia son expresados mediante distintas certificaciones, estructuradas, a su vez, en torno a niveles o escalas (TOEFL, Cambridge, DELF, DALF) o, en otros, a través de los distintos niveles del Marco común europeo de referencia para las Lenguas (Consejo de Europa, 2002), en adelante MCERL. En este sentido, se manifiestan Milton y Alexiou (2009: 197):

$[\ldots]$ if the CEFR has validity as a hierarchy of language level and ability, then each succeeding increase in level in the CEFR should be matched by an increasing demand in the vocabulary knowledge of the learners who take exams at that level. With each progressively higher CEFR band, there should be higher mean scores on vocabulary size measures with groups of learners. If this were not seen then the validity of the CEFR would be called into question. The word lists in the early CEFR materials appear to reflect this pattern. The Threshold (B1) level word 
lists are indeed larger, implying greater knowledge by learners at this level, than the Waystage (A2) material lists.

Ya en 2003, Meara y Milton propusieron una primera aproximación, revisada en 2010 por Milton, en la que la extensión del vocabulario se correspondería con los distintos niveles del MCERL. Para medir el tamaño del vocabulario receptivo, los autores recurrieron al X_Lex (Meara y Milton, 2003), test que mide ítems léxicos independientes presentados sin contexto, en el que se le pide al estudiante que indique aquellas palabras que reconoce o puede usar. La tabla 1 presenta la correspondencia entre el nivel alcanzado del MCERL (CEFR en la tabla, por sus siglas en inglés), la certificación otorgada por Cambridge English y el tamańo del vocabulario, expresado en número de palabras conocidas, según los resultados del test X_Lex. Como el propio Milton (2004) expone el tamaño del vocabulario que se indica es aproximado pues no podemos perder de vista que en la consecución de un determinado nivel hay otras habilidades implicadas, más allá del conocimiento del vocabulario.

\begin{tabular}{|c|l|c|}
\hline CEFR Levels & \multicolumn{1}{|c|}{ Cambridge exams } & X_Lex (5000 max) \\
\hline A1 & Starters, Movers and Flyers & $<1500$ \\
\hline A2 & Kernel English Test & $1500-2500$ \\
\hline B1 & Preliminary English Test & $2750-3250$ \\
\hline B2 & First Certificate in English & $3250-3750$ \\
\hline C1 & Cambridge Advanced English & $3750-4500$ \\
\hline C2 & Cambridge Proficiency in English & $4500-5000$ \\
\hline
\end{tabular}

Tabla 1. Vocabulario aproximado asociado con los niveles del MCERL (Milton, 2010: 224, adaptado de Meara y Milton, 2003: 8).

De nuevo, como ya comentamos para el establecimiento de umbrales léxicos citado anteriormente, las estimaciones realizadas por Meara y Milton se refieren al inglés, lengua favorecida en las investigaciones desarrolladas por los diferentes autores. Sin embargo, el estudio realizado por Milton y Alexiou (2009) resulta ser una excepción por cuanto realizan una cuantificación del tamańo del vocabulario medio equivalente a los diferentes niveles del MCERL, en el que además de comparar los resultados obtenidos entre los distintos niveles, exploran otras lenguas, además del inglés. De este modo, este trabajo nos permite comprobar si existen diferencias en los niveles que se pueden alcanzar según la lengua tomada en consideración, al igual que ocurría con el nivel de cobertura de los textos escritos, que necesitaba de un conocimiento léxico distinto según se tratara del inglés o del francés. Así, Milton y Alexiou (2009) exponen los resultados obtenidos con aprendices de inglés, francés y griego de cuatro países distintos (España, Hungría, Grecia y Gran Bretaña), utilizando las versiones del test X_Lex, que los autores han adaptado a las distintas lenguas. Las medias y desviaciones típicas obtenidas por los estudiantes de los distintos países y lenguas se muestran en la tabla 2. 


\begin{tabular}{|c|c|c|c|c|c|c|}
\hline $\begin{array}{c}\text { CEFR } \\
\text { Level }\end{array}$ & $\begin{array}{c}\text { EFL In } \\
\text { Hungary }\end{array}$ & $\begin{array}{c}\text { EFL in } \\
\text { Greece }\end{array}$ & $\begin{array}{c}\text { French in } \\
\text { Britain }\end{array}$ & $\begin{array}{c}\text { French in } \\
\text { Spain }\end{array}$ & $\begin{array}{c}\text { French in } \\
\text { Greece }\end{array}$ & $\begin{array}{c}\text { Greek in } \\
\text { Greece }\end{array}$ \\
\hline A1 & & $1477(580)$ & & $894(604)$ & $1125(620)$ & $1492(705)$ \\
\hline A2 & & $2156(664)$ & & $1700(841)$ & $1756(398)$ & $2237(538)$ \\
\hline B1 & $3135(434)$ & $3263(434)$ & $952(440)$ & $2194(717)$ & $2422(517)$ & $3338(701)$ \\
\hline B2 & $3668(666)$ & $3304(666)$ & $1882(562)$ & 2450 & $2630(251)$ & $4012(415)$ \\
\hline C1 & $4340(471)$ & $3690(471)$ & & $2675(643)$ & $3212(473)$ & \\
\hline C2 & & $4068(261)$ & $3326(711)$ & $3721(416)$ & $3525(883)$ & \\
\hline
\end{tabular}

$*$ in this field there is only one student score and no SD can be calculated

Tabla 2. Tamaño del vocabulario y MCERL (adaptada de Milton, 2010: 225 y Milton y Alexiou, 2009: 201).

De los resultados presentados en esta tabla se desprenden, al menos, dos observaciones significativas (Milton y Alexiou, 2009), a las que ya aludíamos en un trabajo anterior (De la Maya Retamar, 2015): de un lado, se advierte como la extensión del vocabulario es mayor conforme los alumnos alcanzan niveles superiores del MCERL, existiendo además una relación estadísticamente significativa entre ambas variables -tamaño y niveles-, tal y como lo demuestra el análisis de regresión efectuado. Si en los distintos países el nivel alcanzado por los estudiantes se ve afectado por el conocimiento léxico que poseen, en Espańa este hecho es más notable pues un $67.5 \%$ de la varianza en los niveles del MCERL puede ser explicada por el tamaño del vocabulario. De otro lado, se constata igualmente que existen diferencias entre las distintas lenguas aprendidas en lo que respecta a la extensión del vocabulario necesaria para alcanzar los distintos niveles del MCERL. Así, si prestamos atención al caso del francés y lo comparamos con el inglés, observamos que los resultados obtenidos ponen de manifiesto cómo en francés son necesarias menos palabras para alcanzar cada uno de los niveles del MCERL. De esta forma, mientras que para alcanzar en inglés el nivel B2 es necesario conocer receptivamente una media de 3304 palabras (datos de estudiantes griegos), en francés, son necesarias solo 2630 palabras de media (datos igualmente de Grecia), es decir, 674 palabras de media menos. Hemos de indicar, no obstante, que, en las cifras que se refieren al francés, las medias para un mismo nivel del MCERL presentan mayor variación en los tres países analizados (Espańa, Reino Unido y Grecia) que la que se produce para el inglés, siendo, además, la variación más patente en los datos recogidos en Gran Bretaña. Así, mientras que en España se necesita conocer una media de 2194 palabras en francés para alcanzar el nivel B1, en Grecia esa media es algo superior ( +228 palabras) y en Gran Bretaña sustancialmente menor (-1242 palabras).

Las razones que explican este hecho pueden ser variadas y venir determinadas por las diferencias existentes entre las lenguas analizadas, no solo en lo que respecta a su estructura, sino también por el modo en que se crean y usan las palabras. Así, como indican Milton y Alexiou (2009: 205), siguiendo los planteamientos de Bryson (1990: 6), el inglés dispone de distintas palabras para expresar una misma idea y es una lengua 
en la que, además, existe una diferenciación marcada entre lengua formal e informal, aspecto que no está presente en la lengua francesa. Estas discrepancias, especialmente la idea de que en francés se necesita un caudal léxico menor para alcanzar ciertos niveles, vienen a confirmar las diferencias puestas de manifiesto por Cobb y Horst (2004), respecto del hecho de que la cobertura de textos escritos del francés con las 2000 palabras más frecuentes del vocabulario no se consigue en inglés si a esa misma banda de frecuencia no se le añade el vocabulario académico de la Academic Word List (Coxhead, 2000).

De lo expuesto, concluimos que la extensión del vocabulario es una dimensión que resulta fundamental para los estudiantes, pues, como hemos presentado, no solo está relacionada con la competencia general en lenguas, sino también con la posibilidad de desempeñar efectivamente tareas relacionadas con la comprensión escrita y oral o la expresión escrita. Del mismo modo, como señala De la Maya Retamar (2015), existe una superioridad en los estudios que, analizando esta dimensión, tienen como lengua de referencia el inglés $y$, a pesar de que las conclusiones alcanzadas son de interés para cualquier lengua, se han puesto de manifiesto las diferencias existentes entre lenguas, en relación tanto con los niveles de competencia que podrían alcanzar con un determinado tamańo de vocabulario como los de cobertura de textos. Por este motivo, creemos que es necesario desarrollar nuevos trabajos que permitan, no solo examinar más exhaustivamente estas cuestiones, sino también confirmar los aspectos específicos relativos a la extensión del vocabulario en francés.

\section{Objetivos}

Al objeto de profundizar en el estudio del tamaño del vocabulario receptivo de estudiantes de francés y de su relación con la competencia general en dicha lengua, la investigación que presentamos tiene como objetivos:

1. Medir el tamaño del vocabulario receptivo de estudiantes de francés de Escuela Oficial de Idiomas (EOI en adelante) en un centro extremeño y estudiar el progreso a lo largo de los distintos niveles de las enseñanzas impartidas (de A1 a B2).

2. Establecer una correspondencia entre el tamaño del vocabulario de los estudiantes y los distintos niveles del MCERL.

\section{Metodología de la investigación}

Siguiendo una metodología cuantitativa, se persigue analizar el vocabulario receptivo de los estudiantes encuestados para observar la evolución que se produce a lo largo de los diferentes cursos.

Se trata de un estudio cuasiexperimental, puesto que se ha llevado a cabo en el contexto natural de los sujetos, el aula en la que reciben clases en la EOI. Asimismo, el 
diseño de la investigación es multigrupo, pues se ha trabajado con cuatro grupos distintos y de sólo postest, ya que no se ha pretendido analizar el cambio producido en el vocabulario de los estudiantes tras la intervención docente, sino determinar, una vez recibidas sus clases, el estado de sus conocimientos léxicos.

\subsection{Participantes}

La muestra inicial la constituyeron 67 alumnos de una EOI de Extremadura que se hallaban cursando los cursos de $1^{\circ}$ y $2^{\circ}$ de nivel básico, $2^{\circ}$ de nivel intermedio y $2^{\circ}$ de nivel avanzado, pues son los cursos cuya superación supone alcanzar respectivamente los niveles A1, A2, B1 y B2 del MCERL. No se pudo evaluar el nivel C1 ya que en la Escuela estudiada no se impartía dicho nivel.

Los alumnos se reparten por cursos como se muestra en la siguiente tabla:

\begin{tabular}{|r|c|c|c|c|}
\cline { 2 - 5 } \multicolumn{1}{c|}{} & \multicolumn{2}{c|}{ Nivel básico } & Nivel intermedio & Nivel avanzado \\
\cline { 2 - 5 } & $1 \%(\mathrm{~A} 1)$ & $2 \circ(\mathrm{A} 2)$ & $2 \circ(\mathrm{B} 1)$ & $2 \circ(\mathrm{B} 2)$ \\
\hline Muestra inicial & 20 & 18 & 16 & 13 \\
\hline Aptos en prueba final & 19 & 11 & 10 & 10 \\
\hline No aptos en prueba final & 1 & 7 & 6 & 5 \\
\hline MUESTRA FINAL & & & & 5 \\
Hombres & 8 & 4 & 4 & 0 \\
Mujeres & 11 & 7 & 4 & $\mathbf{1 0}$ \\
\hline NC & 0 & 0 & 2 & $\mathbf{1 0}$ \\
\hline TOTAL & $\mathbf{1 9}$ & $\mathbf{1 1}$ & $\mathbf{1 0}$ & 5 \\
\hline
\end{tabular}

Tabla 3. Caracterización de la muestra

Dado que no todos los alumnos superaron las pruebas conducentes a la obtención de los distintos niveles, la muestra final ha estado compuesta por 50 alumnos de los cuales 30 se encuentran en nivel básico, y 10 en los niveles intermedio y avanzado, respectivamente. Asimismo, como se observa en los datos presentados en la tabla, existe una tendencia a la disminución de alumnos conforme vamos aumentando de curso, por la creciente dificultad de los niveles y las exigencias asociadas a ellos. Es llamativa también la baja matrícula que registra el idioma francés con respecto a otros que se pueden cursar en el mismo centro, como el inglés o el portugués.

En lo que respecta al sexo, la distribución global de la muestra es relativamente equilibrada, aunque la presencia de mujeres $(56,25 \%)$ es algo superior a la de los hombres $(43,75 \%)$. Todos los estudiantes comparten el español como lengua materna a excepción de uno de ellos cuya lengua materna es el catalán. En cuanto a las lenguas extranjeras estudiadas además del francés, 14 no han estudiado otra lengua, 21 conocen otra lengua, inglés en todos los casos, 13 dos lenguas y tan solo 2 conocen 3 y 4 respectivamente. 


\subsection{Instrumentos}

Los alumnos rellenaron un sencillo cuestionario (anexo I) acerca de sus datos personales y detalles sobre sus experiencias con lenguas, tanto en situaciones regladas como en actividades extraescolares.

Asimismo, cumplimentaron el test X_Lex (anexo II) para medir el tamaño de su vocabulario receptivo. Este test fue diseñado por Meara y Milton en 2003 y adaptado al francés en 2006 por Milton. Se trata de un test basado en la frecuencia ya que sus autores asumen que las palabras más frecuentes son las que primero y más fácilmente se aprenden. La elección de este test ha estado determinada por su utilización previa en la mayoría de los escasos estudios que analizan el desarrollo del vocabulario en aprendices de francés (David, 2008; De la Maya Retamar, 2015; De la Maya y Mora, 2019; Graham et al., 2008; Milton, 2006, 2008) lo que nos permitirá realizar comparaciones con los resultados obtenidos.

El test tiene un formato de check-list (Sí/No) en el que al alumno se le presentan 120 palabras, de una en una y debe simplemente marcarlas si son palabras que conoce o puede usar. Del total de palabras incluidas en el test, 100 son palabras reales y 20 son pseudopalabras, es decir, palabras que no existen pero que tienen una construcción similar a la de las palabras verdaderas. Las 100 palabras reales fueron extraídas al azar del listado de frecuencia de Baudot (1992), de modo que 20 pertenecen a las 1000 palabras más frecuentes, otras 20 a las siguientes 1000 palabras frecuentes y así sucesivamente. De esta manera, el vocabulario testado pertenece a las 5000 palabras más frecuentes.

\subsection{Procedimiento}

En un primer momento, los participantes fueron informados sobre los objetivos del estudio, en el que participaron de forma voluntaria, advirtiéndoseles asimismo de la confidencialidad de los datos y del estudio de los mismos con fines exclusivamente de investigación. Posteriormente, fueron evaluados en una única sesión en la que rellenaron el test de vocabulario (anexo II). Se realizó una sola medida de su vocabulario prácticamente a final de curso, momento que consideramos relevante pues ya habían recibido casi la totalidad de horas de instrucción planificadas, lo que nos permitiría comprobar lo aprendido a lo largo del curso académico. Igualmente, se recabó su autorización para acceder a las calificaciones finales ya que, para relacionar los distintos niveles del MCERL con el tamaño de sus vocabularios, solo se podrían tener en cuenta aquellos estudiantes que obtuvieran una calificación de aprobado o superior, calificación que implica la consecución del nivel correspondiente.

\subsection{Resultados}

Para analizar los resultados, hemos realizado una estimación del vocabulario conocido por los estudiantes, contabilizando las respuestas dadas por los alumnos a las palabras verdaderas y restándole las respuestas afirmativas a las pseudopalabras. De esta 
forma se ajusta el resultado final compensando la posible sobreestimación de su vocabulario.

En relación con los resultados obtenidos, hemos de comentar en primer lugar que un alumno de la muestra, que cursaba segundo curso del nivel básico, fue eliminado por contestar afirmativamente a más de 5 pseudopalabras, cifra a partir de la cual se considera que los resultados no son fiables (David, 2008), quedando de este modo la muestra para este test en 49 estudiantes. La media de pseudopalabras de la muestra en su conjunto es de 1,92 , si bien es en los alumnos del curso más alto, $2^{\circ}$ de nivel avanzado, donde la media de estas palabras alcanza la cifra mayor (3,30 de media).

A partir de los datos obtenidos hemos calculado el tamaño ajustado del vocabulario receptivo de los estudiantes (tabla 4), estableciendo la media para cada uno de los niveles de estudio.

\begin{tabular}{|c|c|c|c|c|}
\hline CURSOS & Media & Mínimo & Máximo & Desviación típica \\
\hline A1 & 963,16 & 350 & 2050 & 481,561 \\
\hline A2 & 2420 & 650 & 4450 & 1312,918 \\
\hline B1 & 2470 & 1400 & 3850 & 760,555 \\
\hline B2 & 3105 & 2350 & 3900 & 441,85 \\
\hline
\end{tabular}

Tabla 4. Descriptivos del tamaño del vocabulario receptivo según niveles de estudio

Como pone de manifiesto la tabla anterior y se observa en la gráfica 1, la media de vocabulario aumenta de un nivel al siguiente, siendo en algunos casos el aumento muy llamativo, como el que se produce del nivel A1 al nivel A2 y en otros, del nivel A2 al B1, muy limitado. Es en el nivel A2 en el que las diferencias entre los alumnos son mayores, como lo muestra el dato de la desviación estándar que duplica la de los otros niveles. Así, la diferencia entre el alumno que mejor puntúa y que menos lo hace es de 3800 palabras. Estas variaciones se producen no solo en el interior de ese grupo sino también entre los diferentes grupos ya que, por ejemplo, el alumno que obtiene mejores resultados en el nivel A2, tiene un vocabulario receptivo mayor que los que alcanza la media máxima de vocabulario en los niveles B1 y B2 respectivamente.

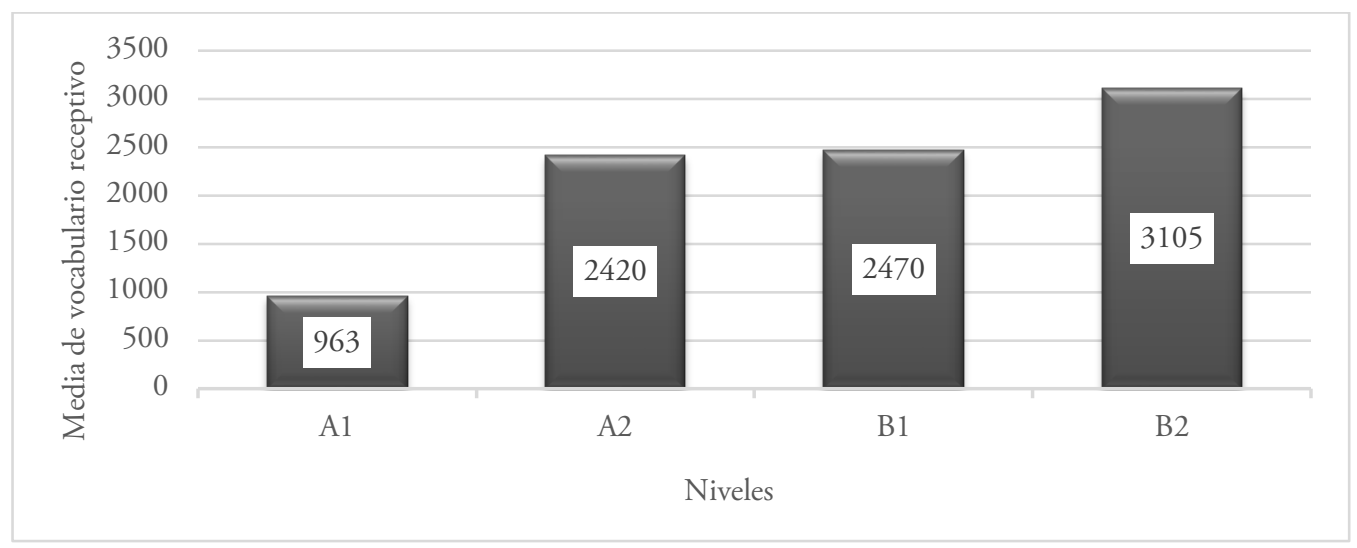

Gráfica 1: Tamaño del vocabulario receptivo según niveles de estudio 
Al objeto de profundizar en los resultados obtenidos, podemos analizar también la media de palabras aprendidas por hora de clase. Para ello, debemos tomar en consideración el número de horas que se imparten en cada curso y la diferencia en el total de palabras de un curso y otro, lo que nos informará del número de nuevas palabras aprendidas. En relación con el número de horas, cada curso, de los seis que imparte la Escuela, recibe una media de 128 horas de clase anuales, por lo que para conseguir los niveles A1 y A2 son necesarias 128 horas respectivamente, mientras que para alcanzar el B1 y el B2 se necesitan 256 en cada caso, ya que son necesarios dos años de estudio por nivel. De este modo, si observamos en detalle los resultados por cursos, nos damos cuenta de cómo, tras el primer ańo de aprendizaje en el que los alumnos aprenden un número considerable de vocabulario (963 palabras), una media de 7,5 palabras por hora de clase, se produce un aumento bastante importante del vocabulario aprendido en el segundo ańo (1457 nuevas palabras), con un media de 11 nuevas palabras por hora de instrucción, seguido de un estancamiento en el nivel B1, con menos de 1 palabra aprendida por hora (50 nuevas palabras), y un remonte final en el nivel B2 con algo más de 600 palabras nuevas aprendidas, casi 2,5 palabras por hora de instrucción.

Con la finalidad de determinar si existen diferencias significativas en el aumento del vocabulario de un nivel al siguiente, una vez verificado el cumplimiento de los supuestos de normalidad, aleatoriedad y homocedasticidad, hemos aplicado la prueba Anova, cuyo resultado $(\mathrm{F}=20,422, \mathrm{p}=, 000)$ nos lleva a rechazar la hipótesis nula $\mathrm{y}$, por lo tanto, la igualdad de las medias entre los distintos niveles. Para determinar entre cuáles de ellos existen diferencias hemos realizado pruebas post-hoc mediante el estadístico de Scheffé, revelándose que las diferencias son significativas solo entre el nivel A1 y los restantes, pero no entre todos los niveles sucesivos. Es decir, que los alumnos no aumentan significativamente de vocabulario de un nivel al inmediatamente superior, lo que no deja de ser sorprendente, rompiendo así la tendencia esperada de un aumento significativo de vocabulario conforme el alumno aumenta de nivel y recibe un mayor número de horas de instrucción.

Finalmente, hemos indagado acerca de la relación existente entre el tamaño del vocabulario receptivo y los distintos niveles del MCERL. El resultado de la prueba de Pearson $(\mathrm{r}=, 715, \mathrm{p}=, 000)$ nos indica que existe una correlación positiva alta, estadísticamente significativa al nivel 0,001 . Puesto que la significatividad es $p=, 000$, hemos llevado a cabo el análisis de regresión lineal simple que nos ha permitido calcular el coeficiente de Determinación. Los resultados $\left(\mathrm{R}^{2}=, 511\right)$ muestran que el $51 \%$ de la variación en los niveles alcanzados viene explicada por el tamańo del vocabulario receptivo.

\section{Análisis y discusión de resultados}

Tal y como hemos expuesto al inicio de la descripción de los datos, tan solo un alumno ha sido excluido de la muestra, al contestar a más de cinco pseudopalabras, 
siendo la media de todos los participantes de 1,92 palabras. El porcentaje de exclusión respecto de esta cuestión es, por tanto, del 2\%, dato que se halla muy por debajo de los estudios consultados que utilizan este tipo de test (David, 2008; De la Maya Retamar, 2015; Mochida y Harrington, 2006; Mora Ramos, 2014, entre otros). Sin embargo, el hecho de que sea un alumno del nivel avanzado el que tenga la media de pseudopalabras mayor de toda la muestra está en la línea de lo afirmado por Eyckmans (2004), quien expone que los alumnos más aventajados no realizan un mejor trabajo distinguiendo las pseudopalabras que sus compañeros con puntuaciones inferiores.

En lo que respecta al tamaño del vocabulario, los resultados obtenidos por los niveles A1 y A2 nos parecen bastante positivos, teniendo en cuenta las horas de instrucción recibidas, 128 en el caso del primero y 256 en el segundo. No existe ningún trabajo que aborde el tamańo del vocabulario receptivo de estudiantes de francés en EOI, por lo que no es posible una comparación de nuestros resultados. Sin embargo, si los comparamos con trabajos realizados en el marco de la educación obligatoria, estos datos son mejores que los de otros estudios realizados con estudiantes de francés de Educación Secundaria en el contexto español. Así, la muestra del estudio de De la Maya Retamar (2015) alcanza una media de 642,5 palabras tras 129 horas de instrucción y la del trabajo de De la Maya y Mora (2019) 611 tras 140 horas. Del mismo modo, son también mejores a los obtenidos por estudiantes de secundaria de francés en el contexto inglés, con similares horas de instrucción recibidas (David, 2008; Milton, 2006) e incluso por estudiantes de últimos niveles de Secundaria y Universidad, en ese mismo contexto educativo, con más del doble de horas de clase recibidas (Graham et al., 2008; Milton, 2008). Es necesario indicar que todos los estudios citados utilizaron como instrumento de medición el text X_Lex, el mismo empleado en este estudio.

Respecto del nivel B1, la extensión de su vocabulario es muy similar a los estudiantes del nivel A2, y aunque no se produce un aumento de vocabulario como sería lógico tras más de 250 horas de estudio, no deja de representar una buena cifra comparada con los resultados obtenidos por las distintas muestras de los estudios antes comentados. El nivel B2 registra una media de vocabulario muy elevada, 3105 palabras, un tamańo de vocabulario suficiente para la cobertura de aproximadamente un $85 \%$ de los textos, según las estimaciones de Cobb y Horst (2004) y la superación de nivel de vocabulario que debe considerarse como objetivo de aprendizaje crucial, de acuerdo con Staehr (2008).

En relación con el desarrollo del vocabulario de un nivel a otro, como hemos visto al analizar la media de vocabulario producido en cada uno de ellos, se manifiesta un incremento del vocabulario de un nivel de estudio al siguiente, de acuerdo con la creencia de que el tamańo del vocabulario aumenta según se incrementa el tiempo de exposición (Webb y Chang, 2012) y se eleva el nivel de estudios (Agustín y Canga, 2016; David, 2008; Milton, 2006; Terrazas y Agustín, 2009). No obstante, este incremento no es significativo entre todos los niveles, a excepción del que se produce del 
nivel A1 al resto de niveles. Este dato concuerda, en lo que respecta a la diferencia entre el nivel A1 y el A2, con los resultados de Canga y Fernández (2014), quienes analizaron también el tamaño del vocabulario receptivo de estudiantes de esos dos niveles de EOI aunque en inglés. Asimismo, está en consonancia con otros estudios, centrados en estudiantes de francés, en los que, analizando más niveles de estudio, los investigadores no encuentran siempre diferencias significativas entre unos niveles y otros (David, 2008; De la Maya y Mora, 2019; Milton, 2006).

A este respecto, lo más llamativo en este análisis sobre el desarrollo es el estancamiento que se produce tras dos ańos, pues del nivel A2 al B1 los alumnos aumentan muy poco su vocabulario. La explicación a esta circunstancia puede deberse a un aspecto que parece caracterizar el desarrollo léxico de estudiantes de francés en el contexto británico (David, 2008; Milton, 2006), que también ha sido evidenciado en los estudios desarrollados por De la Maya y Mora (2019) en el contexto espańol: la existencia de un estancamiento en los primeros años de estudio de este idioma, con un aumento del vocabulario muy poco notable -como describíamos para lo acontecido en el paso del nivel A2 al B1-, seguido por un aumento consistente en la extensión del vocabulario, especialmente notable a partir del tercer año. Las razones del estancamiento citado aún no han sido analizadas en profundidad por ninguna investigación, aunque Milton (2006) expone como probable el rol desempeñado por los materiales didácticos, no solo porque su diseño no responda al objetivo de ampliar el tamaño del vocabulario sino porque, siguiendo a Day (2003), dichos materiales superan la capacidad de comprensión de los alumnos y no aseguran una reutilización constante y significativa del vocabulario que conduzca a un aprendizaje efectivo. Se abre, en este sentido, una línea de trabajo para futuras investigaciones centrada en el análisis de los materiales.

Por otra parte, al confrontar los resultados obtenidos con la propuesta indicativa de Meara y Milton (2003) en la que relacionan las puntuaciones obtenidas con el X_Lex y los niveles del MCERL (Tabla 5), nos damos cuenta de que los resultados para todos los niveles se corresponden con las expectativas formuladas por ambos investigadores, excepto para los niveles B1 y B2 donde se necesita un número menor de palabras para alcanzar el nivel.

\begin{tabular}{|c|c|c|}
\hline CEFR Level & X_Level & Presente estudio \\
\hline A1 & $<1500$ & 963 \\
\hline A2 & $1500-2500$ & 2420 \\
\hline B1 & $2750-3250$ & 2470 \\
\hline B2 & $3250-3750$ & 3105 \\
\hline C1 & $3750-4500$ & -- \\
\hline C2 & $4500-5000$ & -- \\
\hline
\end{tabular}

Tabla 5: Vocabulario aproximado asociado con los niveles de MCERL según Meara y Milton (2003: 8) y comparación con resultados del presente estudio 
Asimismo, si comparamos los resultados obtenidos en cada uno de los niveles del MCERL con los de otros estudios, y de modo más concreto con el realizado por Milton y Alexiou en 2009, en el que el tamaño del vocabulario receptivo de estudiantes de francés es medido mediante el mismo test utilizado en este trabajo, observamos varias cuestiones. De un lado, constatamos el aumento del vocabulario de un nivel a los siguientes, hecho ya reseñado para los resultados de este estudio, confirmando la relación existente entre el tamaño del vocabulario y los distintos niveles del MCERL. Esta afirmación viene apoyada además por los análisis de regresión efectuado mediante el análisis correspondiente que, en el caso de España, muestra cómo, en el estudio de 2009, el 67,5\% de la varianza en los niveles del MCERL puede ser explicada por el tamaño del vocabulario (Milton y Alexiou, 2009) y, en el estudio actual, la varianza es de un $51 \%$, dato que resulta inferior, pero nada desdeńable. A pesar de estas divergencias, $67,5 \%$ en un caso y $51 \%$ en el presente trabajo, estos datos constatan la importancia de la contribución del léxico a la competencia general en lenguas, medida a través de los distintos niveles del MCERL. Si bien existen otras habilidades implicadas más allá del conocimiento del vocabulario, como señalaba Milton (2004), los resultados confirman la relación entre el vocabulario y la competencia en la lengua, planteada por investigadores como Alderson (2005), Fan (2000) o Nasir et al. (2017), entre otros. En segundo lugar, verificamos que las pruebas realizadas en países distintos muestran diferencias en el tamaño de vocabulario que poseen los alumnos para alcanzar los distintos niveles del MCERL (gráfica 2), siendo Grecia aquel en el que sus alumnos presentan un tamaño de vocabulario mayor en prácticamente todos los niveles y Gran Bretańa en el que el tamaño es menor.

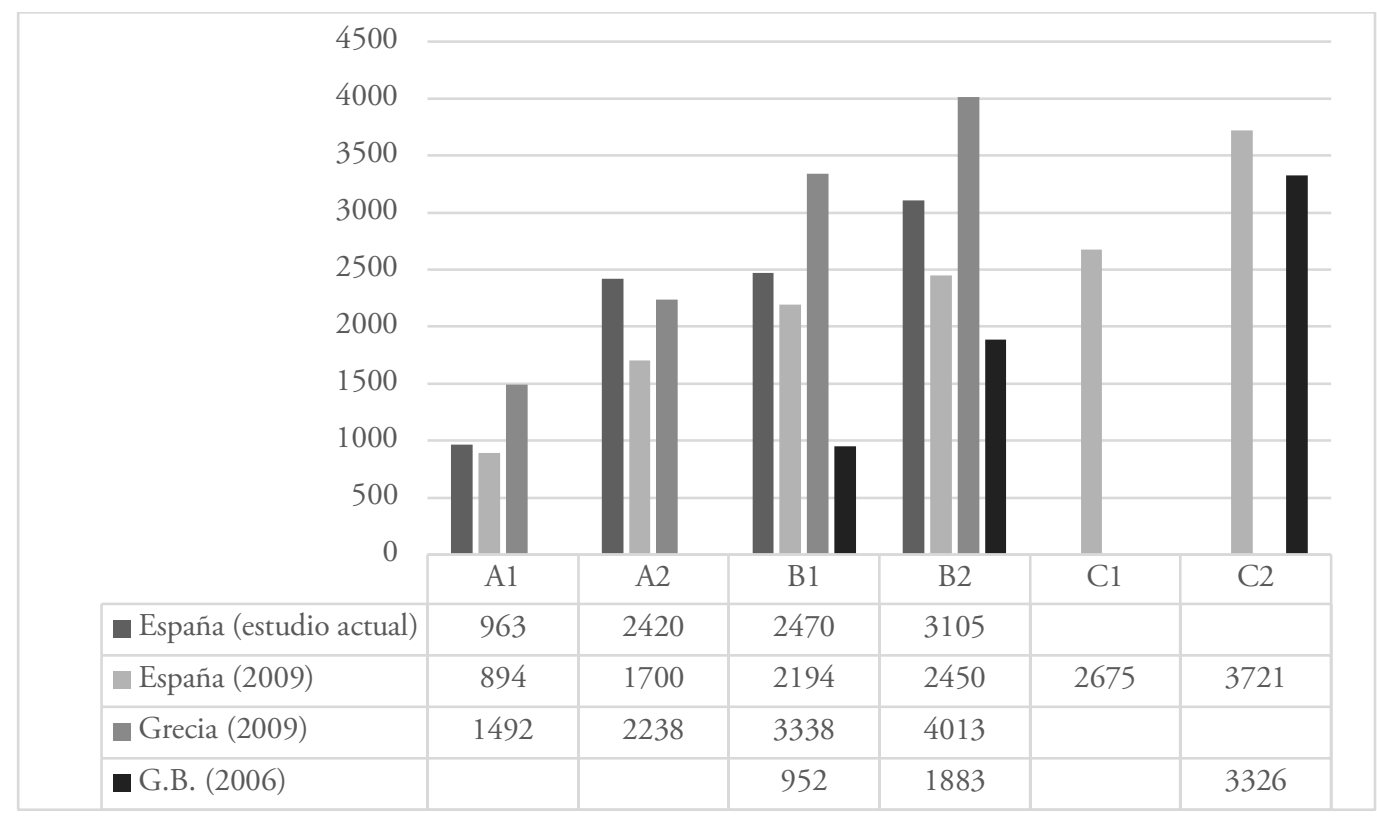

Gráfica 2 Tamaño del vocabulario en francés y niveles del MCERL (adaptada de Milton y Alexiou, 2009: 201) 
Cuando contrastamos los datos referidos a España (2009 y presente estudio) observamos cómo existen diferencias también en el tamaño del vocabulario receptivo, que es mayor en el caso del presente estudio, a excepción del nivel A1 en el que los estudiantes presentan un tamaño de vocabulario similar en ambos trabajos.

Así pues, comparados los resultados, existen diferencias entre los distintos países respecto al tamaño del vocabulario necesario para alcanzar los distintos niveles del MCERL en francés. Aunque es prematuro avanzar una explicación a este hecho, esta puede tener relación con las exigencias curriculares para alcanzar un nivel, no solo entre los distintos países, sino también dentro de un mismo país. Igualmente, las diferencias pueden venir determinadas por los exámenes que permiten acreditar un determinado nivel, de modo que no todos los países sitúan sus exámenes del mismo modo en relación con los distintos niveles, hecho especialmente llamativo en el caso de Gran Bretaña (Milton y Alexiou, 2009). Por todo ello, es necesario la realización de nuevos estudios que permitan, de un lado, ofrecer más datos sobre la extensión del vocabulario en relación con los distintos niveles y de otro, profundizar en las causas que expliquen los resultados aquí obtenidos.

Asimismo, si comparamos los resultados con los obtenidos por Milton y Alexiou (2009) para el inglés (tabla 2), observamos cómo para los niveles A1, B1 y B2, los umbrales de vocabulario necesario para alcanzar esos niveles en francés son inferiores a los del inglés, lo que parece reforzar la idea, ya apuntada, de las diferencias entre lenguas. Es decir, la necesidad de disponer de una extensión de vocabulario mayor en una lengua que en otra para poder alcanzar el mismo nivel de dominio del MCERL.

\section{Conclusiones}

En este trabajo, hemos tratado de arrojar un poco de luz sobre el vocabulario receptivo de estudiantes de francés como lengua extranjera y la relación existente entre su extensión y los distintos niveles del MCERL, tema que, como hemos expuesto, ha sido poco explorado por parte de los investigadores.

En relación con el primer objetivo de nuestra investigación, hemos planteado cuál es la extensión vocabulario receptivo de estudiantes de francés de EOI en un centro extremeńo y analizado el progreso que se produce entre los distintos niveles estudiados (A1 a B2). Si bien se produce un aumento del vocabulario de un nivel al siguiente, las diferencias solo resultan ser significativas del primero (A1) con respecto al resto (A2B1-B2). Se constata igualmente el estancamiento producido tras el segundo año de estudio (A2), fenómeno que también ha sido verificado en otros estudios.

Respecto del segundo objetivo, hemos establecido una correspondencia entre el tamaño del vocabulario de los estudiantes y los distintos niveles del MCERL, constatando no solo que existe una correlación positiva alta entre ambas variables, sino que, además, el 51\% de la variación entre los distintos niveles viene explicada por la extensión del vocabulario receptivo. De ello, se deduce la importancia del vocabulario y la 
necesidad de realizar actividades específicas que permitan desarrollar el vocabulario de nuestros estudiantes.

Finalmente, fruto de la comparación con estudios realizados en otros países y en el propio contexto español, se observan disparidades en la extensión del vocabulario necesaria para superar los distintos niveles. Así, mientras que en España encontramos diferencias, que en el caso más extremo (nivel B2) llegan hasta las 720 palabras, el cotejo con otros países muestra como es Gran Bretaña el país en el que se alcanzan los niveles evaluados con un tamaño del vocabulario menor y Grecia aquel donde la extensión es mayor. Atendiendo a estas conclusiones, planteamos la necesidad de continuar realizando estudios que permitan establecer otras comparaciones, explicar las diferencias y profundizar en el conocimiento acerca del desarrollo léxico de nuestros estudiantes.

\section{REFERENCIAS BIBLIOGRÁFICAS}

ADOLPHS, Svenja \& Norbert SCHMITT (2004): «Vocabulary coverage according to spoken discourse context», in Paul Bogaards y Batia Laufer (ed.), Vocabulary in a second language. Amsterdan, John Benjamins, 39-49.

Agustín, María Pilar \& Andrés CANGA (2016): «Vocabulary growth in young CLIL and traditional EFL learners: evidence from research and implications for education». International Journal of Applied Linguistics, 26:2, 211-227.

Agustín, María Pilar \& Melania TERRAZAS (2009): «Examining the relationship between receptive vocabulary size and written skills of primary school learners». Atlantis, 31:1, 129-147.

Agustín, María Pilar \& Melania TerrazAS (2012): «Vocabulary knowledge development and gender differences in a second language». ELIA: Estudios de Lingüistica inglesa aplicada, 12, 45-75.

ALDERSON, J. Charles (2005): Diagnosing foreign language proficiency: The interface between learning and assessment. Londres, Continuum.

ARNAUD, Pierre \& Henri BÉJOINT [ed.] (1992): Vocabulary and applied linguistics. Basingstoke, Macmillan.

BATISTA, Roselene \& Marlise HORST (2016): «A New Receptive Vocabulary Size Test for French». The Canadian Modern Language Review / La Revue canadienne des langues vivantes, 72:2, 211-233.

BAUDOT, Jean (1992): Fréquences d'utilisation des mots en français écrit contemporain. Montreal, Les Presses de l'Université de Montréal.

BLAIS, Isabelle (2014): La taille du vocabulaire et les habiletés réceptives des étudiants adultes de niveau 6 en Francisation. Tesis de maestría, Universidad de Quebec, Montreal. URL: http://www.archipel.uqam.ca/6673/1/M13471.pdf.

BOGAARDS, Paul (1994): Le vocabulaire dans l'apprentissage des langues étrangères. Paris, Didier. 
BOGAARDS, Paul \& Batia LAUfER [ed.] (2004): Vocabulary in a second language. Amsterdan, John Benjamins B.V.

CAlaQUe, Élizabeth \& Jacques DaVID [ed.] (2004): Didactique du lexique. Contextes, démarches, supports. Bruselas, De Boeck Université.

CANGA, Andrés \& Almudena FERNÁNDEZ (2014): «Motivation and L2 receptive vocabulary knowledge of Spanish EFL learners at the Official School of Languages». Miscelánea: a Journal of English and American Studies, 49, 13-28.

ClARK, Eve (1993): The lexicon in acquisition. Cambridge, Cambridge University Press.

COADY, James \& Thomas HUCKIN [ed.] (1997): Second language vocabulary acquisition. Cambridge, Cambridge University Press.

COBB, Tom \& Marlise HORST (2004): «Is there room for an academic word list in French?», in Paul Bogaards y Batia Laufer (eds.), Vocabulary in a second language: selection, acquisition and testing. Amsterdan, John Benjamins, 15-38.

CONSEJO DE EUROPA (2002): Marco común europeo de referencia para las lenguas: aprendizaje, enseñanza, evaluación. Madrid, MECD y Anaya.

COXHEAD, Averil (2000): «A new academic word list». TESOL Quarterly, 34:2, 213-238.

DALler, Helmut; James MilTON \& Jeanine TreFFers-DALler [ed.] (2007): Modelling and assessing vocabulary knowledge. Cambridge, Cambridge University Press.

DAVID, Anabelle (2008): «Vocabulary breadth in French L2 learners». The Language Learning Journal, 36:2, 167-180.

DE LA MAYA RETAMAR, Guadalupe (2015): El desarrollo de la competencia léxica en la adquisición del francés como lengua extranjera en la ESO. Tesis doctoral dirigida por Rafael Alejo González y María Rosa Luengo González. Badajoz, Universidad de Extremadura. URL: http://dehesa.unex.es/handle/10662/4045?locale-attribute=pt.

DE LA MAYA RETAMAR, Guadalupe \& Inmaculada MORA RAMOS (2019): «Estudio del conocimiento léxico en FLE de estudiantes españoles de Secundaria». Revista Complutense de Educación, 30:2, 527-543.

EYCKMANS, June (2004): Measuring receptive vocabulary size. Utrecht, LOT.

FAN, May (2000): «How big is the gap and how to narrow it? An investigation into the active and passive vocabulary knowledge of L2 learners». RELC Journal, 31:2, 105-119.

FERNÁNDEZ, Almudena (2017): «Individual and contextual variables in EFL receptive vocabulary: analysis of gender effect and motivation of CLIL primary graders». Odisea. Revista de estudios ingleses, 15, 57-72.

GALISSON, Robert (1971): Inventaire thématique et syntagmatique du français fondamental. París, Hachette.

GrAHAM, Suzanne; Brian RiChARDS \& David MALVERN (2008): «Progress in learning French vocabulary in a one-year advanced course at school». Journal of French Language Studies, 18:3, 349-364. 
GREIDANUS, Tine. \& Lydius NIENHUIS (2001): «Testing the quality of word knowledge in a second language by means of words associations. Types of distractors and types of associations». The Modern Language Journal, 85:4, 567-577.

GREIDANUS, Tine; Bianca BEKS \& Richards WAKELY (2006): «Testing the development of French word knowledge by advanced Dutch-and English-speaking learners and native speakers». The Canadian Modern Language Review/La Revue canadienne des langues vivantes, 62:4, 509-532.

Grossmann, Francis; Anne-Marie PaVeau \& Gérard PetiT [ed.] (2005): Didactique du lexique: langue, cognition et discours. Grenoble, ELLUG.

GYLLSTAD, Henrik (2013): «Looking at L2 vocabulary knowledge dimensions from an assessment perspective -challenges and potential solutions», in Camilla Bardell; Christina Lindqvist \& Batia Laufer (ed.), L2 vocabulary acquisition, knowledge and use: new perspectives on assessment and corpus analysis. Amsterdan, EUROSLA, 11-28.

HiLton, Heather (2007): «La compétence lexicale en production orale», in Actes du colloque DILTEC, recherches en acquisition et en didactique des langues étrangères et secondes. París, Université Sorbonne Nouvelle - Paris 3, 1-10.

HARKIO, Noora \& Päivi P. PIETILÄ (2016): «The role of vocabulary breadth and depth in reading comprenhension: a quantitative study of Finnish EFL learners». Journal of Language teaching and research, 7:6, 1079-1088.

JIMÉNEZ, Rosa María [ed.] (2010): Gender perspectives on vocabulary in foreign and second languages. Basingstoke, Palgrave Macmillan.

JIMÉNEZ, Rosa María [ed.] (2014): Lexical availability in English and Spanish as second language. Dordrecht, Springer.

JiMÉNEZ, Rosa María \& Andrés CANGA (2019): «El léxico disponible en inglés de chicos y chicas españoles adolescentes». ELIA: Estudios de Lingüistica inglesa aplicada, 19, 157 176.

JIMÉNEZ, Rosa María \& Soraya MORENO (2004-2005): «Promoting English vocabulary research in primary and secondary education: Test review and test selection criteria». ES. Revista de Filología inglesa, 25, 171-187.

JIMÉNEZ, Rosa María \& Melania TERRAZAS (2005-2008): «The receptive vocabulary of English foreign language young learners». Journal of English Studies, 5-6, 173-191.

LAUFER, Batia (1989): "What percentage of text-lexis is essential for comprehension?», in Christer Laurén \& Marianne Nordman (ed.), Special Language: from humans thin-king to thinking machines. Clevedon, Multilingual Matters, 316-323.

LAUFER, Batia (1992): «How much lexis is necessary for reading comprehension? ", in Pierre J.L. Arnaud \& Henri Béjoint (ed.), Vocabulary and applied linguistics. Londres, Macmillan, 126-132.

LAUFER, Batia \& Geke C. RAVENHORST-KALOVSKI (2010): «Lexical threshold revisited: Lexical text coverage, learners' vocabulary size and reading comprehension». Reading in a Foreign Language, 22:1, 15-30. 
LAUFER, Batia; Cathie ELDER; Kathryn Hill \& Peter CONGDON (2004): «Size and strength: do we need both to measure vocabulary knowledge?». Language Testing, 21:2, 202226.

LINDQVIST, Christina; Camilla BARDEL \& Anna GUDMUNDSON (2011): «Lexical richness in the advanced learner's oral production of French and Italian L2». Iral, 49, 221-240.

MEARA, Paul (1990): «A note on passive vocabulary». Second Language Research, 6:2, 150-154.

MEARA, Paul (1996): "The dimensions of lexical competence», in Gillian Brown, Kirsten Malmkjaer \& John Williams (ed.), Performance and competence in second language acquisition. Cambridge, Cambridge University Press, 35-53.

MeArA, Paul \& James Milton (2003): X_Lex. The Swansea levels test. Newbury, Express.

MEARA, Paul (2009): Connected words: word association and second language vocabulary acquisition. Amsterdan, John Benjamins.

MELKA, Francine (1997): «Receptive vs. productive aspects of vocabulary», in Norbert Schmitt \& Michael McCarthy (ed.), Vocabulary description, acquisition and pedagogy. Cambridge, Cambridge University Press, 84-102.

MiLTON, James (2004): «Testing yours students vocabulary size and what this can tell you», in ANUPI: Second International Congress Towards greater professionalization in language teaching. México, ANUPI. URL: http://www.anupi.org.mx/PDF/04007_JamesMilton.pdf

MiLTON, James (2006): «Language lite? Learning French vocabulary in school». French Language Studies, 16, 187-205.

MiLTON, James (2008): «French vocabulary breadth among learners in the British school and university system: comparing knowledge over time». Journal of French Language Studies, 18:3, 333-348.

MiLTON, James (2010): «The development of vocabulary breadth across the CEFR levels», in Inge Bartning; Maisa Martin y Ineke Vedder (ed.), Communicative proficiency and linguistic development: intersections between SLA and language testing research. EUROSLA, 211-232.

MiLTON, James (2013): «Measuring the contribution of vocabulary knowledge to proficiency in the four skills», in Camille Bardel; Christina Lindqvist \& Batia Laufer (ed.), L2 vocabulary acquisition, knowledge and use. EUROSLA, 57-78.

MiLtON, James \& Thomai ALEXIOU (2009): «Vocabulary size and the common european framework of reference for languages», in Brian Richards; Hellmut M. Daller; David Malvern; Paul Meara; James Milton \& Jeanine Treffers-Daller (ed.), Vocabulary studies in first and second language acquisition. Basingstoke, Palgrave, 194-211.

MiLTON, James; Jo WADE \& Nikola HOPKINS (2010): «Aural word recognition and oral competence in a foreign language», in Rubén Chacón Beltrán; Christian Abelló Contesse \& M. Mar Torreblanca López (ed.), Insigths into non-native vocabulary teaching and learning. Bristol, Multilingual Matters, 83-98.

MOCHIDA, Kira \& Michael HARRINGTON (2006): «The Yes/No test as a measure of receptive vocabulary knowledge». Language Testing, 23:1, 73-98. 
MORA RAMOS, Inmaculada (2014): Análisis del tamaño de vocabulario receptivo en alumnos AICLE y no AICLE de $\sigma^{\circ}$ de Primaria. Trabajo Fin de Máster inédito. Badajoz, Universidad de Extremadura.

NASIR, Nur Fatima; Nor Ashikin MANAN \& Noraziah AZIZAN (2017): «Examining the relationship between vocabulary knowledge and general English language proficiency». ESTEEM Journal of Social Sciences and Humanities, 1, 15-22.

NATION, Paul (1990): Teaching and learning vocabulary. Nueva York, Newbury House Publishers.

NATION, Paul (2001): Learning vocabulary in another language. Cambridge, Cambridge University Press.

NATION, Paul (2006): «How large a vocabulary is needed for reading and listening?». The Canadian Modern Language Review, 63:1, 59-82.

NATION, Paul (2016): Making and using word list for language learning and testing. Amsterdan, John Benjamin.

ÖZTÜRK, Meral (2003): «Lexical competence in the Common European Framework of Reference for Languages", ponencia presentada en el I International Symposium on the Common European Framework and foreign language education in Turkey. Bursa. URL: https://docsbay.net/lexical-competence-in-the-common-european-framework-ofreference-for-languages.

Ovtcharov, Valentin; Tom COBB \& Randall HALTER (2006): «La richesse lexicale des productions orales: mesure fiable du niveau de compétence langagière». The Canadian Modern Language Review, 63(1), 107-125.

QIAN, David D. (2002): «Investigating the relationship between vocabulary knowledge and academic reading performance: an assessment perspective». Language Learning, 52(3), 513-536.

READ, John (2000): Assessing vocabulary. Cambridge, Cambridge University Press.

RICHARDS, Brian James; David MALVERN \& Suzanne GRAHAM (2008): «Word frequency and trends in the development of French vocabulary in lower-intermediate students during year 12 in English schools». The Language Learning Journal, 36:2, 192-213.

SCHMITT, Norbert (2010): Researching vocabulary: a vocabulary research manual. Houndmills, Palgrave Macmillan.

STAEHR, Lars Stenius (2008): «Vocabulary size and the skills of listening, reading and writing». The Language Learning Journal, 36:2, 139-152.

TERrAZAS, Melania \& María Pilar AGUSTín (2009): «Exploring the increase of receptive vocabulary knowledge in the foreign language: a longitudinal study». International Journal of English Studies, 9:1, 113-133.

TIBDAlL, Françoise \& Jeanine, TREFFERS-DALLER (2008): Analysing lexical richness in French learner language: what frecuency list and teacher judgements can tell us about basic and advanced words. Journal of French Language Studies, 18 :3, 299-313. 
TREVILLE, Marie Claude (2000): Vocabulaire et apprentissage d'une langue seconde. Outremont (Québec), Les Éditions Logiques.

TREVILle, Marie Claude \& Lise DuQueTte (1996): Enseigner le vocabulaire en classe de langue. París, Hachette.

WeBB, Stuart Alexander \& Anna CHANG (2012): «Second language vocabulary growth». RELC Journal, 43:1, 113-126.

ZAREVA, Alla; Paula SCHWANENFLUGEL \& Yordanka NiKOlOVA (2005): «Relationship between lexical competence and language profiency. Variable sensitivity». Studies in Second Language Acquisition, 27, 567-595.

\section{ANEXO I}

\section{CUESTIONARIO SOBRE EL PERFIL GENERAL}

Estimados alumnos/as: mediante este cuestionario se pretende conocer algunos aspectos relativos a vuestra trayectoria en el aprendizaje de lenguas extranjeras, que nos serán útiles para explicar qué aspectos pueden influir en el desarrollo del vocabulario del francés. Estas respuestas serán tratadas de forma anónima y con fines exclusivamente científicos. Asimismo, al objeto de equiparar el vocabulario receptivo que poseéis con los niveles de MCERL, os pedimos vuestra autorización para comprobar a final de curso si habéis alcanzado el nivel que estáis cursando.

Gracias por vuestra colaboración

\section{DATOS PERSONALES}

Nombre:

Apellidos:

Edad:

Sexo: Curso:

\section{EXPERIENCIAS CON LENGUAS}

- Lengua Materna:

- ¿Estudias o has estudiado otros idiomas además del FRANCÉS? SÍ__ NO_

\begin{tabular}{|l|l|l|}
\hline IDIOMA & DURACIÓN (años) & NIVEL ALCANZADO (A1, A2, B1...) \\
\hline & & \\
\hline & & \\
\hline & & \\
\hline
\end{tabular}




\section{ANEXO II}

Test de vocabulario receptivo $X \_L e x$ (Meara, 2006)

Nombre: Apellidos:

Curso: Sexo: Fecha:

Por favor, lee las siguientes palabras. Algunas de estas palabras son palabras francesas reales y otras son palabras inventadas haciendo que parezcan reales. Marca las palabras que conoces o que puedes usar. Observa el siguiente ejemplo:

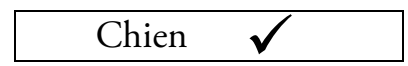

Gracias por tu ayuda

\begin{tabular}{|l|l|l|l|l|l|}
\hline De & Distance & Abattre & Absurde & Achevé & Manchir \\
\hline Aussi & Intellectuel & Argument & Résolution & Habitation & Vernique \\
\hline Jamais & Oreille & Teinte & Lame & Voler & Gillais \\
\hline Long & Contrôle & Publication & Juré & Financer & Ultimation \\
\hline Plusieurs & Quantité & Congruence & Salarié & Lassitude & Talenté \\
\hline Regarder & Catégorie & Sportif & Exploiter & Soupçon & Satisfactoire \\
\hline Lequel & Maximum & Agiter & Voulu & Cracher & Brigeable \\
\hline Divers & Futur & Équipage & survivre & Prêcher & Reparlance \\
\hline Début & Tromper & Baser & Pistolet & Coiffé & Houroux \\
\hline Solution & Respect & Coutume & Entamer & Indignation & Provocatif \\
\hline Genre & Résistance & Insecte & Analogie & Sonde & Spirité \\
\hline Existence & Solide & Tournée & Consommateur & Valve & Porvent \\
\hline Possibilité & Douter & Domestique & Défi & Pelouse & Slendre \\
\hline Structure & Entretenir & Pot & Guérir & Réflexe & Touceul \\
\hline Révéler & Bombe & Panneau & Séduire & Attachement & Statutoire \\
\hline Source & Acteur & Déplacement & Antiquité & Débrouiller & Rescuer \\
\hline Participer & Aile & Trésor & Entrevue & Équivaloir & Aperne \\
\hline Soudain & Étoile & Étonné & Objection & Localement & Vicinité \\
\hline Style & Baisser & Outil & Pourcent & Opportun & Introis \\
\hline Procédé & Spécialement & Financement & Taxi & Serpent & Siève \\
\hline
\end{tabular}

